\title{
Use of Space-Filling Curves for Additive Manufacturing of Three Dimensionally Varying Graded Dielectric Structures Using Fused Deposition Modeling
}

Zachary Larimore*a, Sarah Jensen ${ }^{\mathrm{a}}$, Paul Parsons ${ }^{\mathrm{a}}$, Brandon Good ${ }^{\mathrm{b}}$, Kelsey Smith $^{\mathrm{b}}$ and Mark Mirotznik ${ }^{\mathrm{a}}$

a: Electrical and Computer Engineering Department, University of Delaware, Newark, DE 19716 USA emails: larimore@udel.edu,pparsons@udel.edu and mirotzni@udel.edu

b: Naval Surface Warfare Center, Carderock Division, West Bethesda, MD 20817-5700 USA emails: brandon.good@navy.mil and kelsey.m.smith@navy.mil

* Denotes Corresponding Author 


\begin{abstract}
In this paper the authors present a novel design tool for realizing dielectric structures with spatially varying electromagnetic properties via additive manufacturing (AM). To create tool paths ideal for AM processes, space-filling curves were utilized. Using fused deposition modeling (FDM), spatially varying structures were printed that produced a spatially varying relative permittivity. A wide range of varying fill fractions were printed and evaluated, demonstrating good agreement between the simulated and measured results. Furthermore, the authors verified that this design tool can be applied to practical structures by designing, printing and testing a gradient index flat lens.
\end{abstract}




\section{Introduction}

Electromagnetic (EM) structures with spatially graded dielectric properties have a variety of practical applications including passive beam steering, graded index lenses (GRIN) [1] and antireflective surfaces [2]. However, fabricating structures with complicated EM properties that vary in three dimensions is challenging. To achieve varying EM properties, most investigators have employed subtractive manufacturing techniques such as computer numerically-controlled (CNC) milling. This approach creates an array of subwavelength voids within a solid dielectric substrate $[3,4]$ producing spatially varying effective dielectric properties. However, while useful for two-dimensional designs, this method is difficult to extend to structures that vary in three dimensions. Moreover, the large number of voids that would be required could compromise the part's mechanical robustness. A relatively new fabrication technique that is well suited for realizing three dimensionally varying dielectrics is additive manufacturing (AM). AM can create complex 3D structures that are not possible using traditional subtractive methods [5].

Previous efforts towards realizing 3D graded structures using AM have centered on the unit cell geometry shown in Figure 1. This architecture consists of a series of solid dielectric blocks of varying sizes connected by thin lines [6]. If the imensions of the blocks and distances between adjacent blocks are much smaller than the material wavelength (i.e., $\Lambda<<\lambda$ ) then the effective dielectric properties will be proportional to the local volume fraction of printed material to background material (normally air). While effective, this design methodology has some undesirable characteristics. One is its need for support material. For complex structures, removing support material is typically non-trivial, even with further development and proliferation of watersoluble support materials. Another potential disadvantage stems from the interconnected cube geometry being developed for polymer jet printing. While polymer jetting enables the fabrication of fine features, such as those shown in

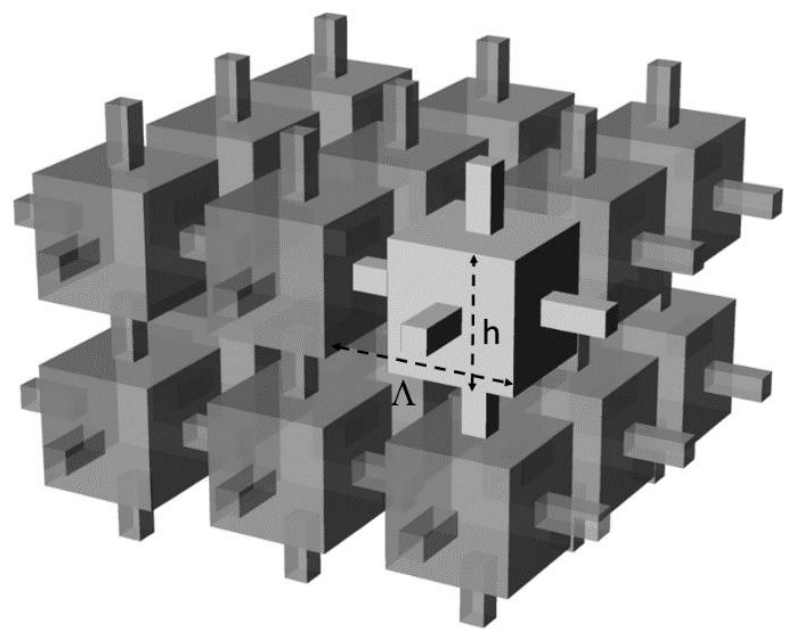

Figure 1: Previously published geometry for creating spatially varying permittivity distribution using additive manufacturing [6]. In this method a distribution of interconnected dielectric cubes is printed to realize a part with spatially varying effective EM properties.

Figure 1, it also requires the use of UV curable polymers. These materials display relatively high EM loss and may mechanically degrade over time [7].

Thus, this paper describes a new approach for generating 3D graded EM structures that exploits the properties of space-filling curves. This method was developed with fused deposition modeling (FDM) in mind. FDM is a much more prevalent and cost effective technology than polymer jetting, and it allows the use of very low loss thermoplastics with robust mechanical characteristics. In the sections that follow, the authors first describe the mathematical properties of the space-filling curves as well as 
the relationship of the geometry to the local effective dielectric properties. The fabrication process was then detailed which was used for both calibration and validation experiments. Lastly, the application of this new methodology was demonstrated by designing, fabricating and experimentally characterizing a graded index lens at microwave frequencies.

\section{Methods}

\subsection{Space-filling Curves}

Peano and Hilbert originally described space-filling curves in the late $19^{\text {th }}$ century $[8,9]$. Their general characteristic is that they are continuous and can completely fill higher dimensional spaces. Over the years, a number of different space-filling curves have been studied and have been used for a variety of applications ranging from image processing to antenna design. For this application, the researchers chose a Peano type curve based specifically on the geometry described in Regazzoni [10] and illustrated in Figure 2a. The figure demonstrates that by varying the number of turns, N, of a continuous curve the user can control the local fraction of printed material to background material and thus can locally define an effective permittivity. Each curve in Figure 2 a represents a single unit cell, or pixel, in this design approach. This particular geometry was chosen for two main reasons. First, since the curves have equal lengths of material oriented in both the $\mathrm{x}$ and $\mathrm{y}$ directions, the resultant EM properties are isotropic for EM fields polarized in the $x-y$ plane. Second, for each unit cell, the curves can be oriented so the beginning and ending points always occur in the same corners (e.g., bottom left and top left, respectively). This allows unit cells to be connected end-to-end in columns and rows, creating a single continuous curve with a spatially varying volume fraction of material (Fig. 2b). This single continuous curve minimizes the total number of starts and stops needed during the printing process, an optimal approach for print speed, resolution, and resulting mechanical strength. Since layers of these space-filling curves are stacked in the $\mathrm{z}$ direction to fabricate the final 3D part, the 3D graded structure requires a single tool path with no need for support material. A potential disadvantage of this approach, demonstrated in more detail in the next section, is that fields polarized in the $\mathrm{z}$ direction will interact with a different effective permittivity than fields polarized in the $x-y$ plane. This is due to the difference in material volume when viewed in the z-direction vs. the $\mathrm{x}-\mathrm{y}$ plane. This is a flaw that is intrinsic to this type of design and printing. Thus, the effective permittivity will be slightly anisotropic. 


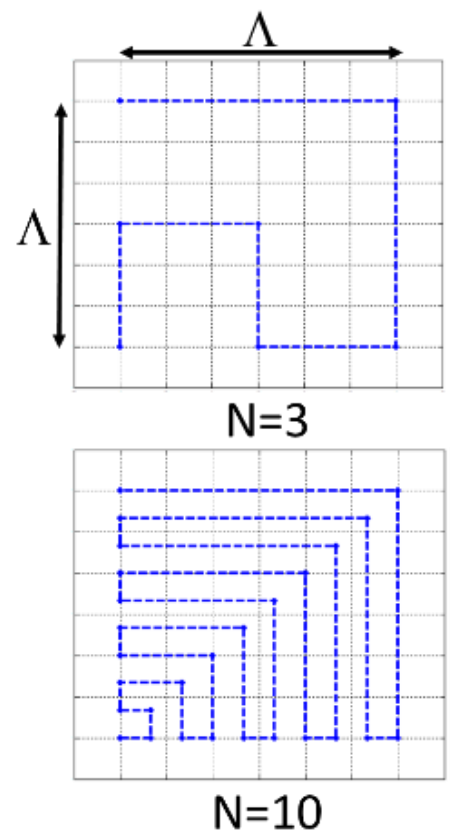

(a)

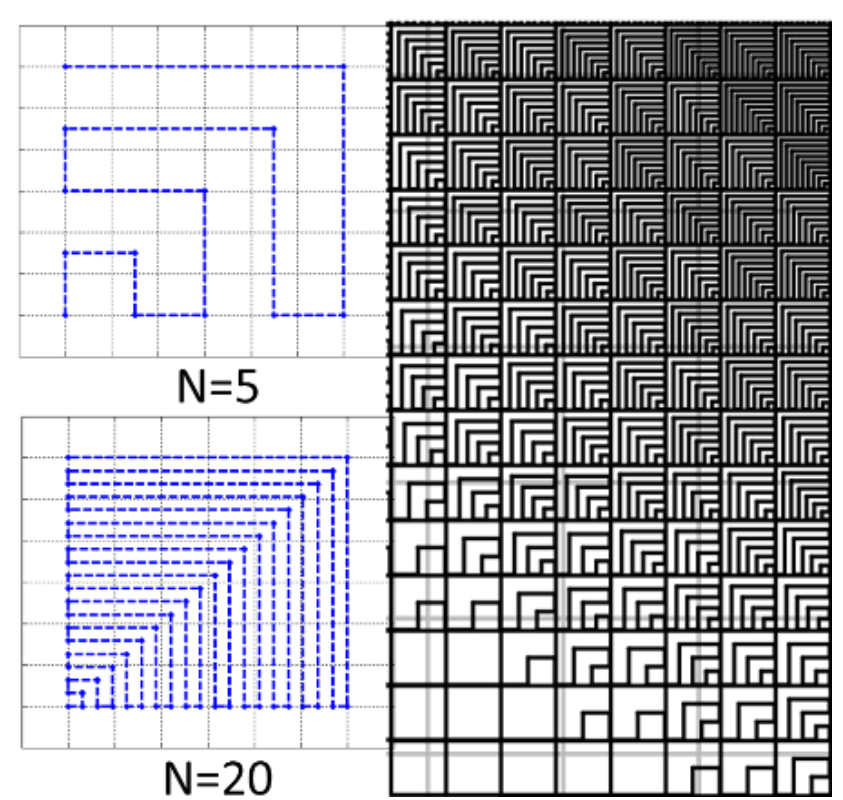

(b)

Figure 2: (a) Illustration showing the space-filling curve geometry used to create spatially varying permittivity distributions. By varying the order $N$, the user can tune the local volume fraction of printed material and thus the local effective permittivity. (b) Illustration showing how the unit cell geometries can be connected end-to-end resulting in a single continuous curve with spatially varying effective EM properties.

\subsection{Modeling of the Effective Anisotropic Permittivity of the Unit Cell}

Before designing graded 3D structures, it is first necessary to understand how the patterning influences the effective dielectric properties of the unit cell. When the unit cells (Figure 2a) are much smaller than the material wavelength (i.e., $\Lambda<<\lambda$ ), the EM properties can be approximated using effective media theory (EMT). EMT approximates the permittivity of an inhomogeneous composite as an effective quantity that varies as a function of its geometry and the constituent material properties [11]. For simple geometries EMT produces analytical mixture formulas, however, for the space-filling curves shown in Figure 2a, it requires a more computational approach. Thus, a numerical scheme for predicting the effective dielectric properties of the unit cell was developed.

Figure 3 illustrates a FDM printed unit cell in more detail. The printed filament has a cross sectional area (top) given by

$$
A_{\text {cross }}=h(W-h)+\pi\left(\frac{h}{2}\right)^{2}
$$

where $\mathrm{W}$ and $\mathrm{h}$ denote the diameter of the extrusion nozzle and the thickness of each printed layer, respectively. The volume fraction, VF, of printed polymer material to the total volume of the unit cell is then given by

$$
V F=\frac{A_{\text {cross }} \cdot L_{\text {tot }}}{\Lambda^{2} \cdot h}
$$


where $\Lambda^{2}$ is the area of the unit cell and $\mathrm{L}_{\text {tot }}$ is the total length of printed material per unit cell. For these geometries, $\mathrm{L}_{\mathrm{tot}}$ is related to the space-filling curve parameter, $\mathrm{N}$, by $\mathrm{L}_{\mathrm{tot}}=(\mathrm{N}+1) \Lambda$. Substitution gives the volume fraction of printed material within a single unit cell as a function of the geometry of the space-filling curve and the printed filament shape:

$$
V F=\frac{\left(W-\left(1-\frac{\pi}{4}\right) h\right) \cdot(N+1)}{\Lambda}
$$

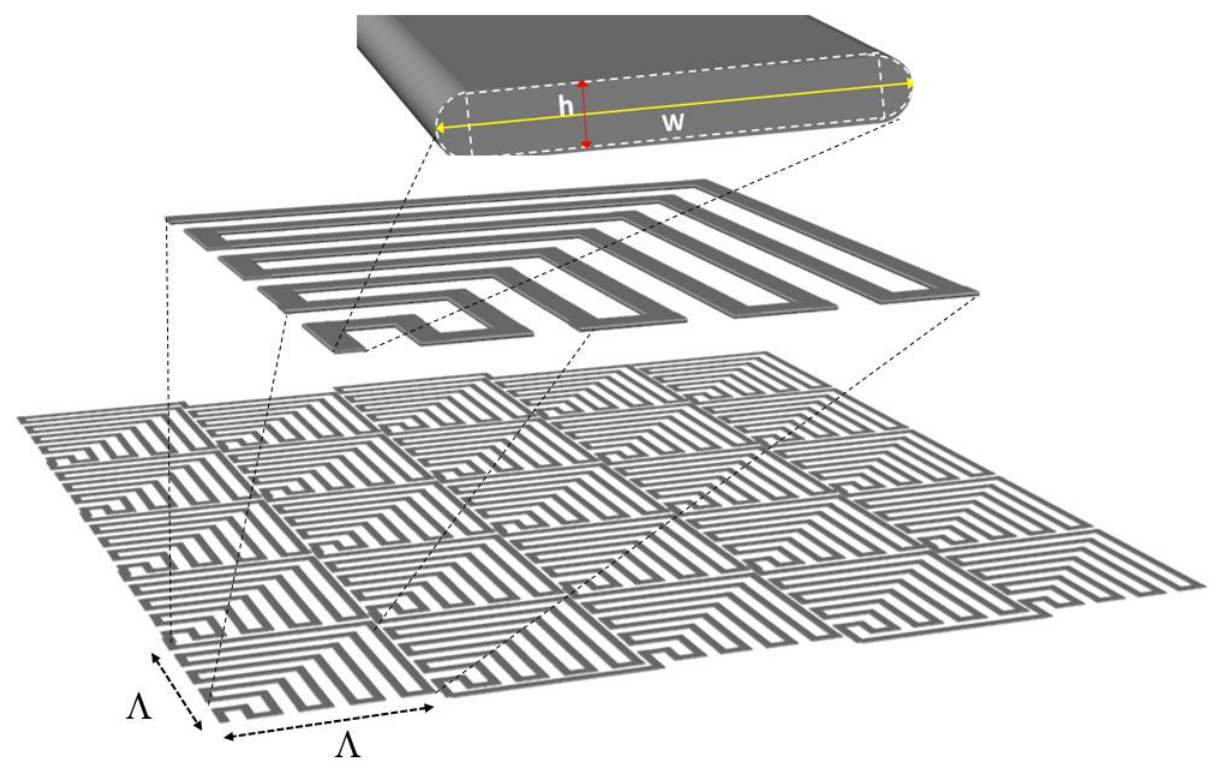

Figure 3: More detailed illustration of a space-filling curve geometry printed via FDM. The cross sectional shape of a printed polymer filament is shown at the top.

To compute the effective permittivity tensor as a function of volume fraction, the algorithm described in Figure 4a was implemented. In this approach, the authors solved two different EM problems. The first is a rigorous solution for a plane wave incident on the complex space-filling geometry depicted in Figures 3 and $4 \mathrm{~b}$. For these calculations, it was assumed the unit cell periodically repeated in both the $\mathrm{x}$ and $\mathrm{y}$ directions. Using a custom developed rigorous coupled wave algorithm (RCWA) solver [12], the researchers computed the complex reflection and transmission coefficients as a function of unit cell geometry (e.g., $\mathrm{N}, \mathrm{h}, \mathrm{W}$ and $\Lambda$ ), incident field parameters (e.g., wavelength, polarization and incident angle) and permittivities of the printed polymer and background materials. The second problem, shown in Figure 4c, is a homogenous anisotropic slab that has the same thickness, $\mathrm{d}$, as the geometry in Figure $4 \mathrm{~b}$. For this case, simple analytical expressions to compute the complex reflection and transmission coefficients were found [13].

To determine the effective permittivity of the space-filling curve geometry, an iterative optimization algorithm was used to vary the anisotropic permittivity of the slab until the difference in transmission and reflection between the actual geometry (Fig. 4b) and the slab problem (Fig. 4c) were 
minimized. It was found that a pattern search optimization algorithm converged quickly towards the optimal solution. This process was repeated three times to obtain the permittivity along each of the primary axes (i.e., $\varepsilon_{\mathrm{x}}, \varepsilon_{\mathrm{y}}$ and $\varepsilon_{\mathrm{z}}$ ) depicted in Figures $4 \mathrm{~b}$ and $4 \mathrm{c}$. Specifically, to find $\varepsilon_{\mathrm{x}}$ and $\varepsilon_{\mathrm{y}}$, the authors illuminated the structure with normally incident plane waves polarized along the $\mathrm{x}$ or $\mathrm{y}$ axes, respectively. To determine $\varepsilon_{\mathrm{z}}$, the researchers illuminated with an obliquely incident TM polarized plane wave that has a reasonably large z-component of the electric field. Typically, the incident angle was set at $60^{\circ}$ from the normal direction.

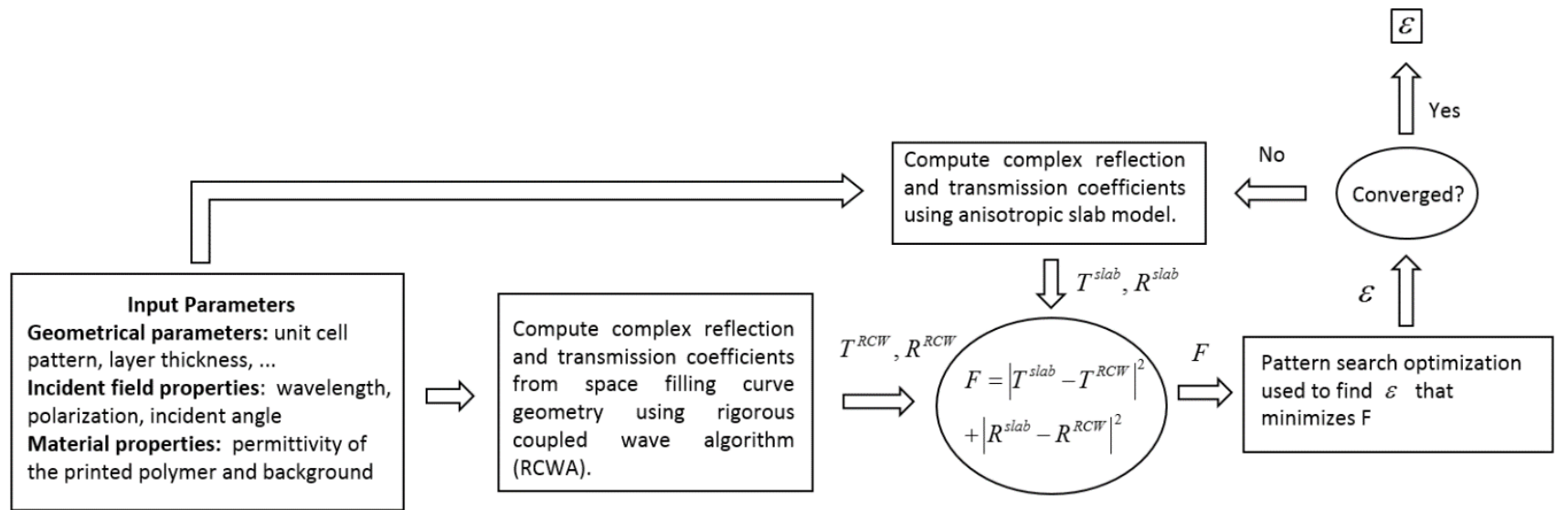

(a)

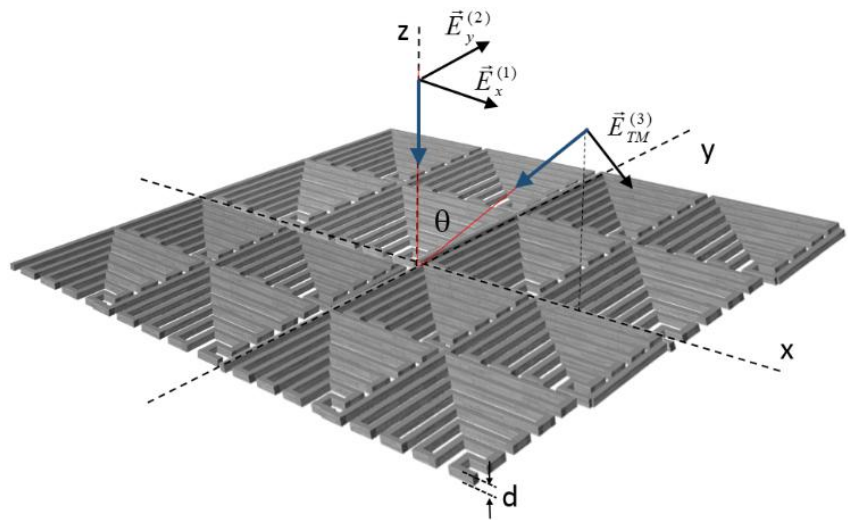

(b)

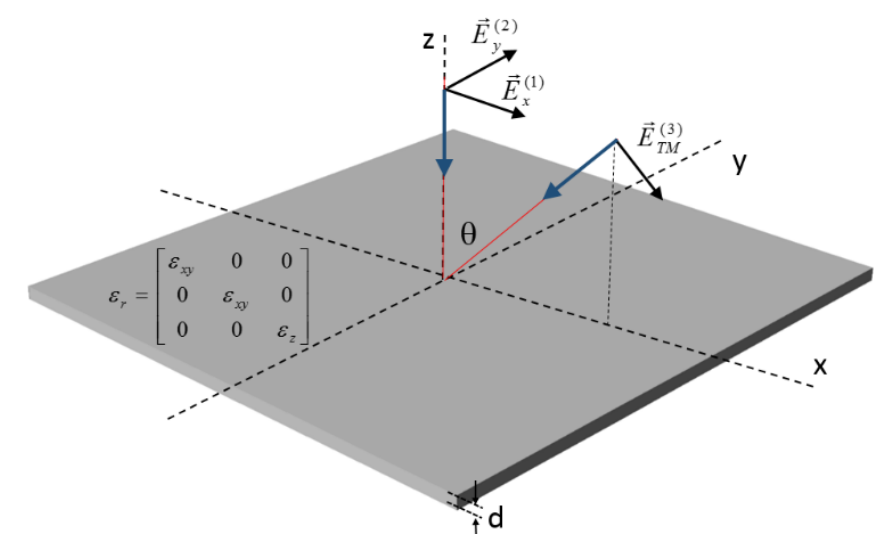

(c)

Figure 4: (a) Flow diagram describing the computational algorithm developed to extract the effective anisotropic permittivity tensor of the space-filling curve geometry. The method employs an iterative optimization scheme that varies the anisotropic properties of a homogenous slab $(c)$ until the reflection and transmission properties match the solution acquired using the RCWA method on the actual spacefilling curve geometry $(b)$.

Using the algorithm described above, the authors calculated the effective permittivity tensor for the geometry shown in Figure 3 for a range of geometrical parameters and material properties. Figure 5 illustrates an example of the predicted effective permittivity as a function of volume fraction. For this example, the polymer was a polycarbonate $\left(\varepsilon_{\mathrm{r}}=2.68\right)$ and the background material was air. The extruded filament diameter, $\mathrm{W}$, layer height, $\mathrm{h}$, and unit cell size, $\Lambda$, were fixed at $0.3 \mathrm{~mm}, 0.125 \mathrm{~mm}$ and 3.0 
$\mathrm{mm}$, respectively. The volume fraction was varied by changing the order of the space-filling curve $\mathrm{N}$. As expected, the components of the permittivity tensor along the $\mathrm{x}$ and $\mathrm{y}$ direction (i.e., $\varepsilon_{\mathrm{x}}$ and $\varepsilon_{\mathrm{y}}$ ) were found to be equal. However, at any given volume fraction, the through-thickness component, $\varepsilon_{\mathrm{z}}$, was smaller than the transverse components. The asterisks in Figure 5 denote measured values, showing good agreement between measured and computed values.

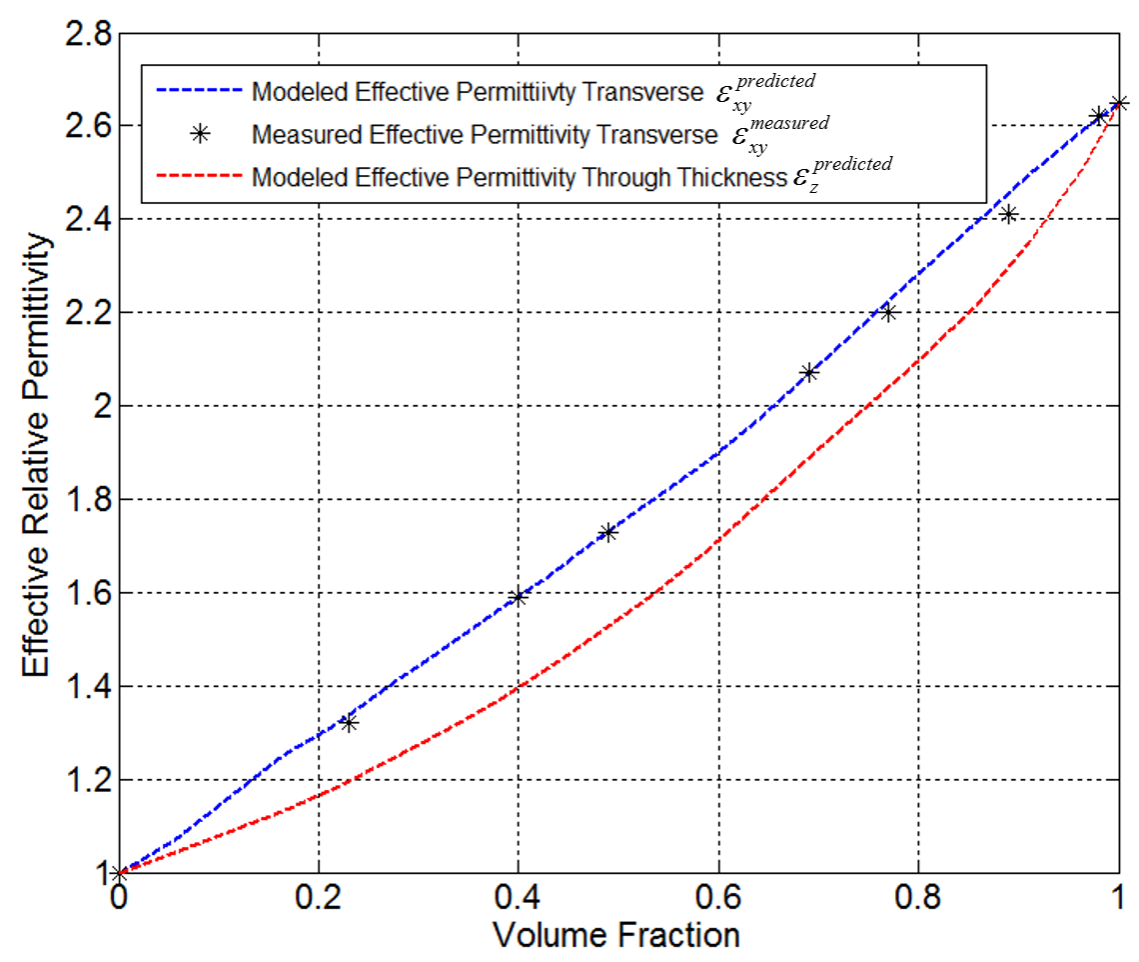

Figure 5. The predicted effective relative permittivity of a space-filling curve as a function of the volume of printed material relative to the unit cell volume (i.e., volume fraction). The blue curve denotes the effective properties in the transverse plane, while the red curve denotes the effective properties in the through-thickness direction. The asterisks indicate measured values.

\subsection{Design Approach for 3D Graded Structures}

To design and fabricate three-dimensionally varying dielectric structures, the authors employed the methodology illustrated in Figure 6. The process begins with a desired 3D permittivity distribution that has been sectioned along the z-axis into thin planar layers (Fig 6a). The thickness of each layer depends on the width of the nozzle used to print and is typically less half the diameter of the nozzle, and for the printer and nozzle used is typically $0.125 \mathrm{~mm}$. The unit cell can be varied somewhat, but is limited less than half the nozzle diameter. The continuous permittivity distribution of each layer is then sampled on a two-dimensional grid in which the grid spacing equals the unit cell length, $\Lambda$. Within each unit cell the local volume fraction, $\mathrm{VF}$, of material required to match the desired local permittivity is calculated using the EMT model described in the previous section. Once the volume fraction distribution is determined, the exact parameters for the space-filling curve (i.e., N) can be found using Eq. [3] (Fig. 6b). Custom Matlab ${ }^{\mathrm{TM}}$ code then generates the tool path needed to realize the continuous space-filling 
curve for each layer (Fig. 6c). It should be noted that the z-direction permittivity is graded by changing printing pattern from layer to layer per the z-direction permittivity described in Figure 5, an example of this can also be seen in Figure 8. Lastly, the structure is printed layer-by-layer using FDM producing a mechanically robust structure with the desired 3D permittivity distribution (Fig. 6d). It should also be noted that each unit cell has a border which the space filling curve intersects with which allows for high fill fraction layers to be printed onto low fill fraction layers without compromising structural integrity.

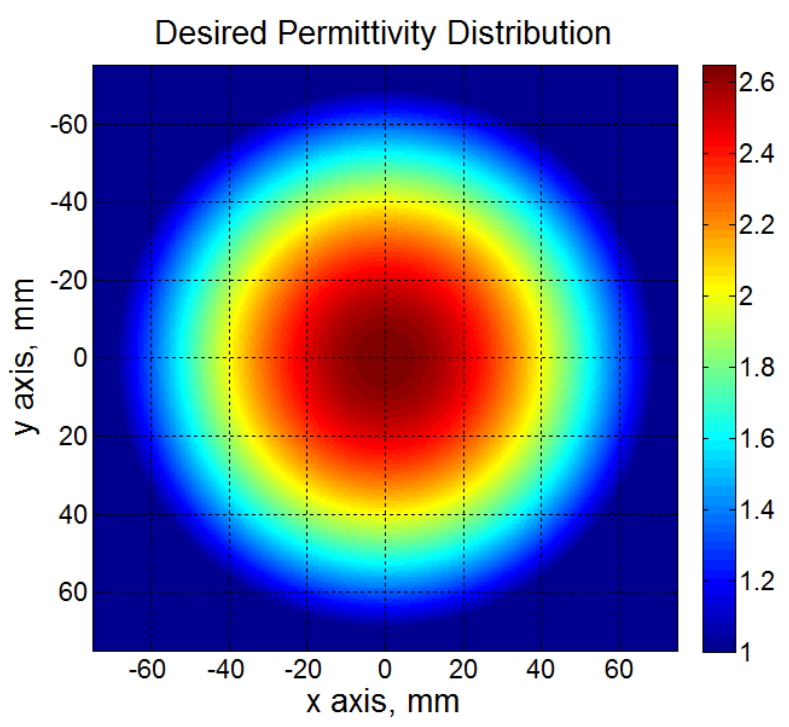

(a)

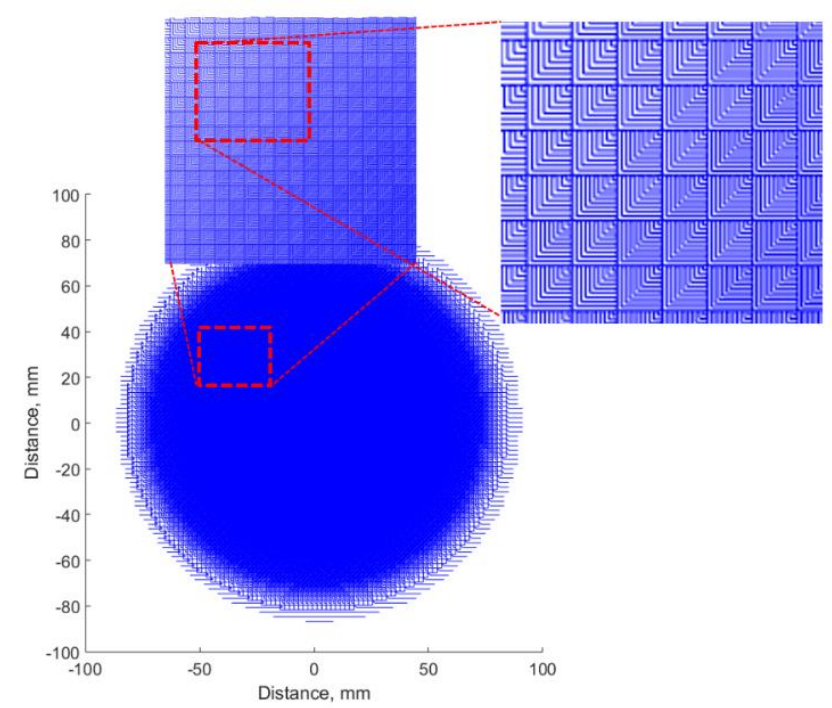

(c)

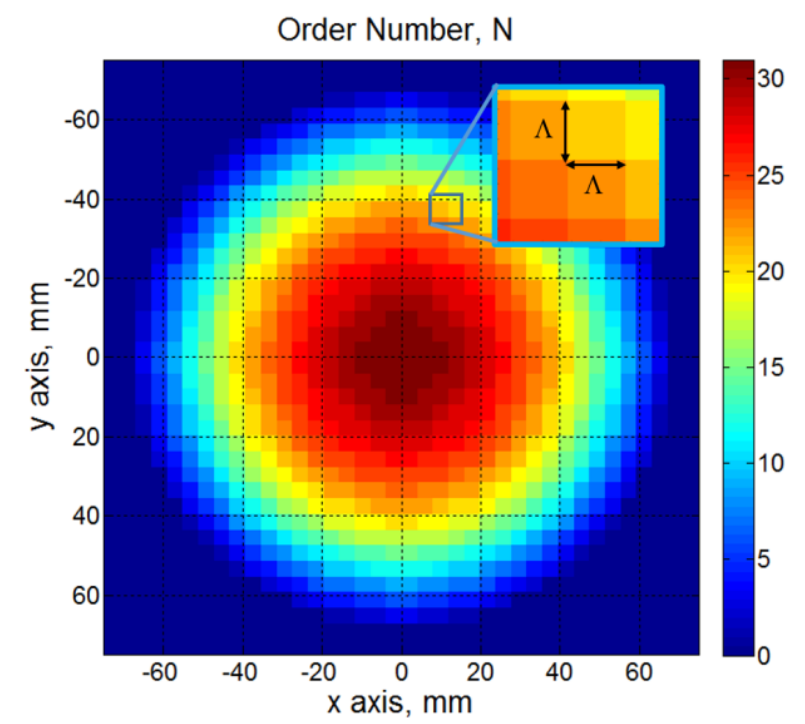

(b)

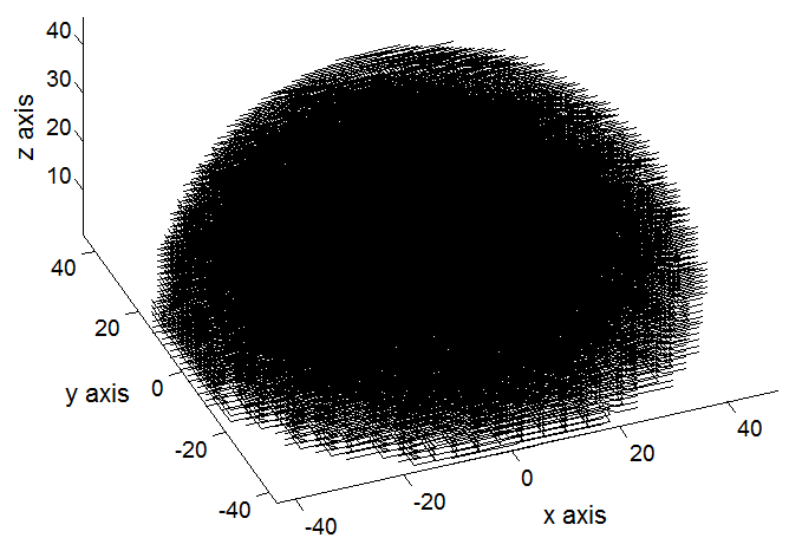

(d)

Figure 6. Illustration of design approach to fabricating 3D graded structures. (a) Desired 3D permittivity distribution is sliced into thin sections along the z-axis. (b) The continuous permittivity distribution for each slice is sampled onto a $2 \mathrm{D}$ array of unit cells. Within each unit cell, a spacefilling curve of order $N$ is found that most closely approximates the desired local permittivity. (c) Unit cell curves for a given layer are connected end-to-end resulting in a single continuous toolpath. $(d)$ Each slice is printed layer-by-layer producing a mechanically robust $3 D$ part. 


\subsection{Fabrication}

For fabrication the nScrypt $\mathrm{t}^{\mathrm{TM}}$ 3Dn-300 system shown in Figure 7 was used. This is a dual deposition system with one head capable of depositing custom and commercial inks and pastes via micro dispensing, and the other head capable of extruding polymers (FDM). For this application, the researchers only required the FDM print head. The nScrypt is also outfitted with a fiducial alignment camera, a 3D laser scanning system, and a 300x300 mm heated print bed. The nScrypt system can print lines as narrow as $20 \mu \mathrm{m}$ with stages that are capable of maintaining a positional accuracy within $<1.0$ $\mu \mathrm{m}$.

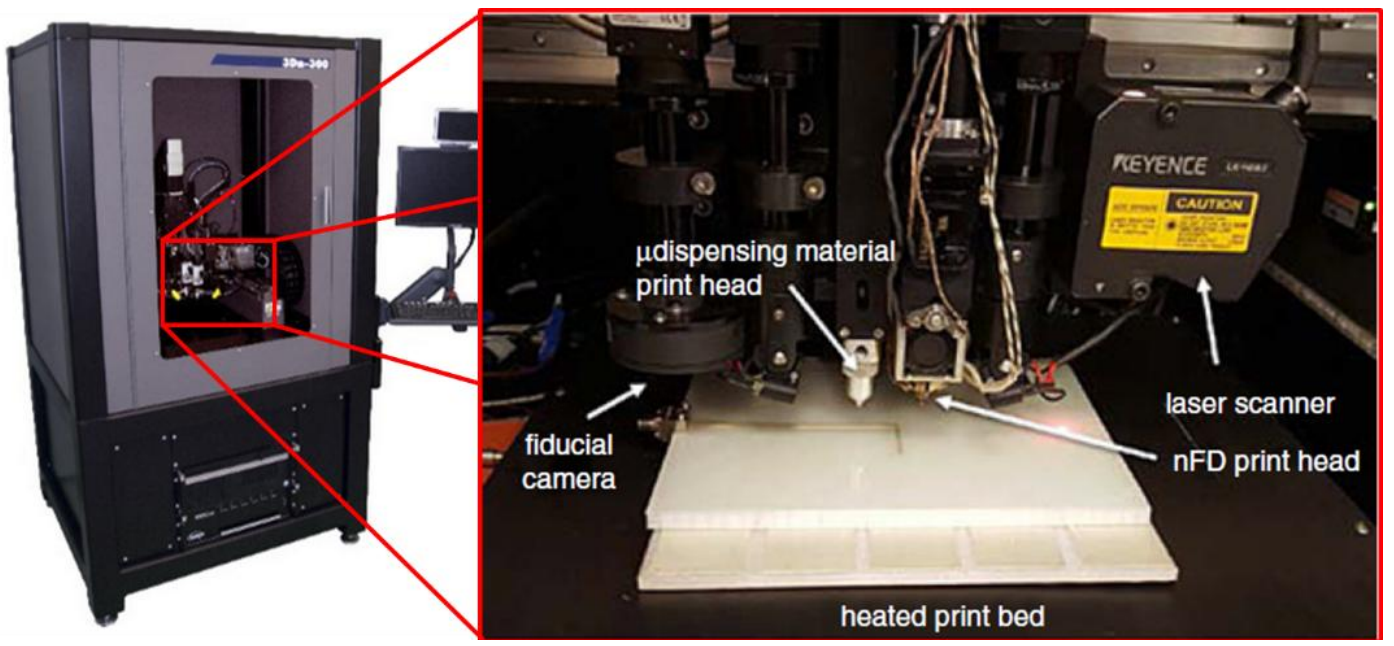

Figure 7: Fabrication was performed using an nScrypt 3Dn-300 system.

For FDM printing the chosen thermoplastic was a polycarbonate obtained from matterhackers.com that displayed excellent EM properties and mechanical strength. Prior to printing, the authors performed EM characterization of this polycarbonate over a wide band of frequencies (8-40 $\mathrm{GHz}$ ), and found that the material was non-dispersive with a dielectric constant of $\varepsilon_{\mathrm{r}}=2.68$ and a loss $\operatorname{tangent}$ of $\tan \delta=0.0005$. In comparison, most UV curable polymers used for polymer jetting have loss tangents in excess of $\tan \delta>0.02$ [7]. Polymer extrusion was performed at a nozzle temperature of $295^{\circ} \mathrm{C}$ and a print bed temperature of $130^{\circ} \mathrm{C}$. The nozzle was a $300 \mu \mathrm{m}$ inner diameter nTip ${ }^{\mathrm{TM}}$, and deposition occurred at $60 \mathrm{~mm} / \mathrm{s}$ with layer thicknesses of $125 \mu \mathrm{m}$. Figure 8a shows a printed Luneburg lens structure with a micro-CT scan showing the printed microstructure of the space-filling curve. 


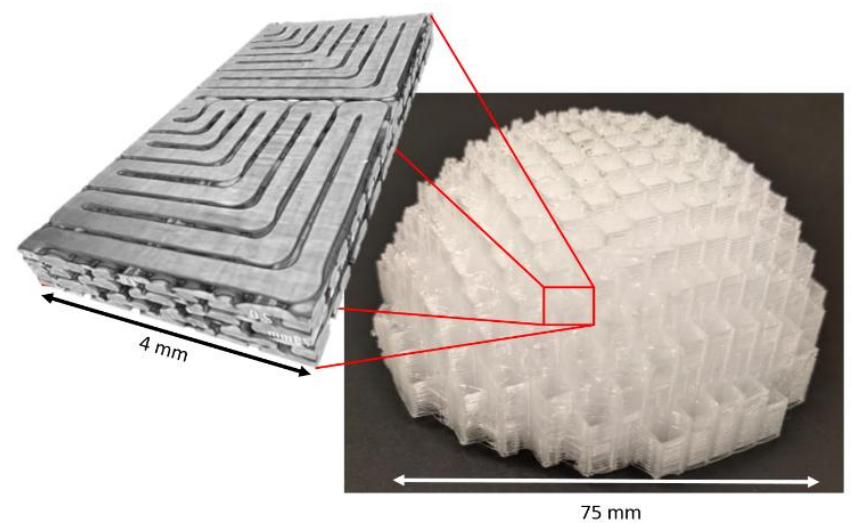

(a)

Figure 8. (a) 3D structure designed to have the permittivity distribution shown in (b). The magnified image in (a) was obtained using micro-CT imaging and shows the printed microstructure of the unit cell and space-filling curve.

\section{Results}

3.1. Experimental Validation of the EMT Model

To validate the EMT model, the researchers fabricated a series of seven calibration samples. Each sample, printed using the nScrypt FDM print head, was $200 \mathrm{~mm}$ x $200 \mathrm{~mm}$ x $1.0 \mathrm{~mm}$ in size and was designed with a uniform volume fraction of material (i.e., same unit cell geometry throughout). However, the unit cell geometry was varied from sample to sample by changing the space-filling curve order, N, from 3 to 17 . This corresponded to material volume fractions ranging from $23 \%$ to $98 \%$. A custom-built free-space focused beam system (Figure 9) was used to measure the dielectric constant of each sample across the frequency range of $4 \mathrm{GHz}$ to $40 \mathrm{GHz}$. To evaluate any anisotropy in the xyplane, the part was rotated $90^{\circ}$ and the measurement repeated. The results show that the measured effective permittivities in the transverse direction (i.e., $\varepsilon_{x y}$, asterisks in Figure 5) closely agree with the properties predicted by the EMT model. The authors note that they did not measure the component of permittivity in the through-thickness direction $\left(\varepsilon_{z}\right)$; however, given the accuracy of the predictions of the transverse components, the authors are confident that the full EMT is providing accurate results. 


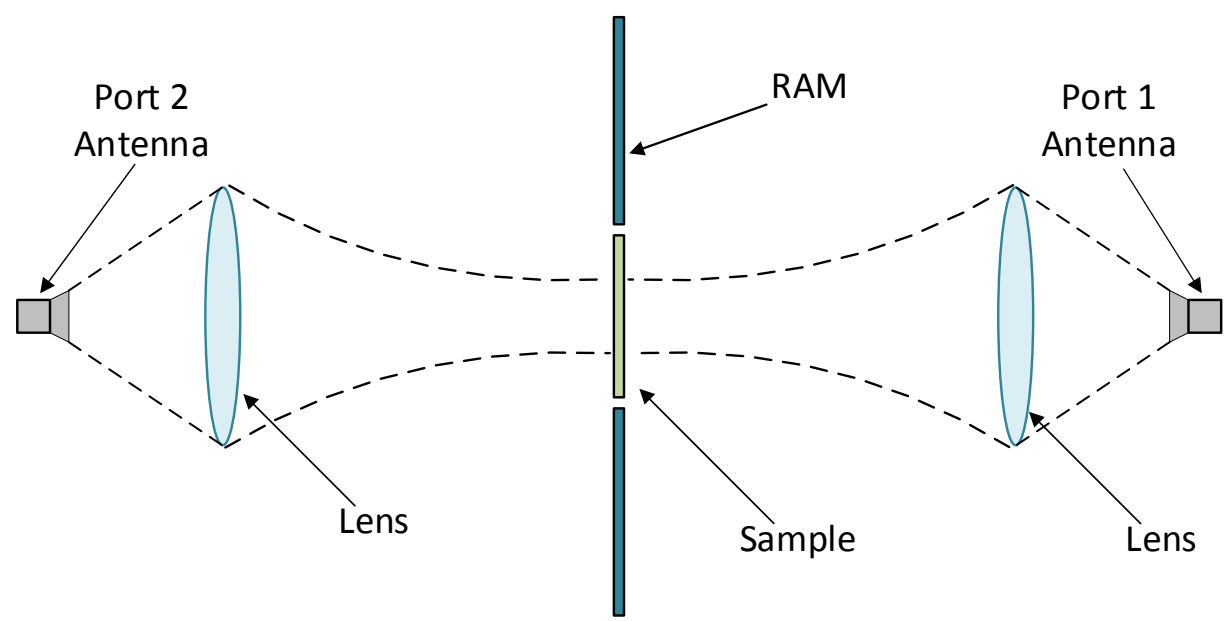

Figure 9: Free space anechoic chamber setup for measuring transmission characteristics of fabricated samples.

\subsection{Example \#1: Grid Pattern with Spatially Varying Permittivities}

To validate the ability to create spatially varying dielectric properties using space-filling curves, the researchers designed and fabricated the simple grid geometry shown in Figure 10. For this sample, a $200 \mathrm{~mm}$ x $200 \mathrm{~mm}$ x $8.0 \mathrm{~mm}$ plate was divided into a 6 × 6 grid of square cells. Within each cell the order, $\mathrm{N}$, of the space-filling curve was varied from $\mathrm{N}=0$ to $\mathrm{N}=104$ as shown in Figure 10a. The sample was FDM printed (Fig. 10b) by extruding polycarbonate filaments $\left(\varepsilon_{\mathrm{r}}=2.68\right) 0.3 \mathrm{~mm}$ in diameter and $0.125 \mathrm{~mm}$ in height. Sixty-four layers were required to print the $8.0 \mathrm{~mm}$ thick sample. The predicted effective permittivity for each cell, shown in Figure 10a, was calculated using the effective media model previously described. The authors experimentally validated the predictions using the characterization system shown in Figure 11. Here, a $30 \mathrm{GHz}$ plane wave at normal incidence illuminated the sample, and a small antenna probe scanned across the backside of the sample to measure the complex transmission coefficient at each point using a vector network analyzer (i.e., Agilent E8364B PNA). From this data the transmitted phase, averaged across each cell, was calculated and compared to predicted values (Figs. 10c and 10d). The authors found that the measured and predicted transmission phases closely agreed over the entire sample with the highest errors occurring for cells with the lowest permittivity values. At the lower fill fractions the distance between printed lines is large enough that fabrication errors start to increase the error in measured permittivity values. 


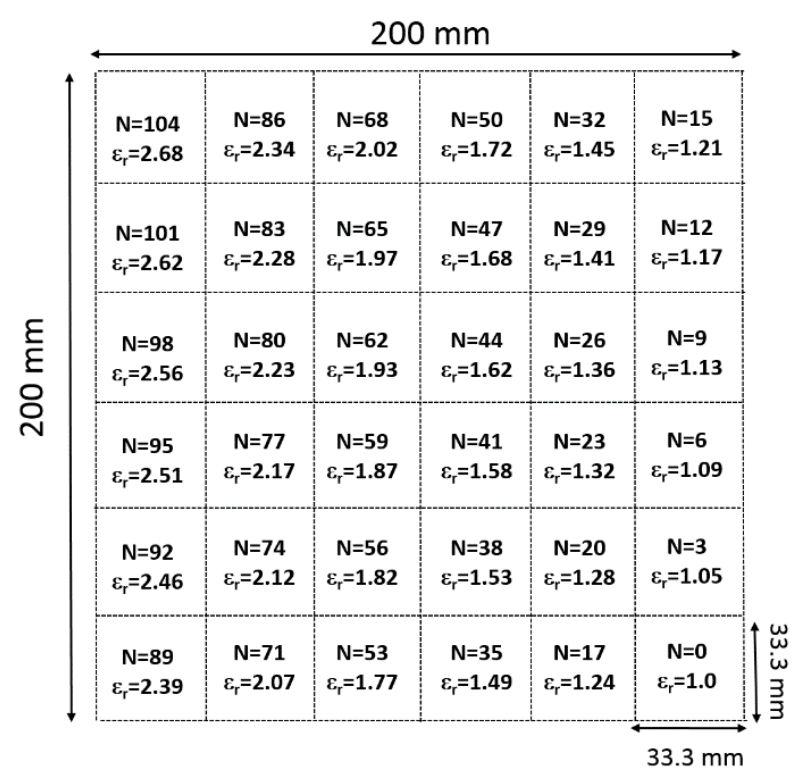

(a)

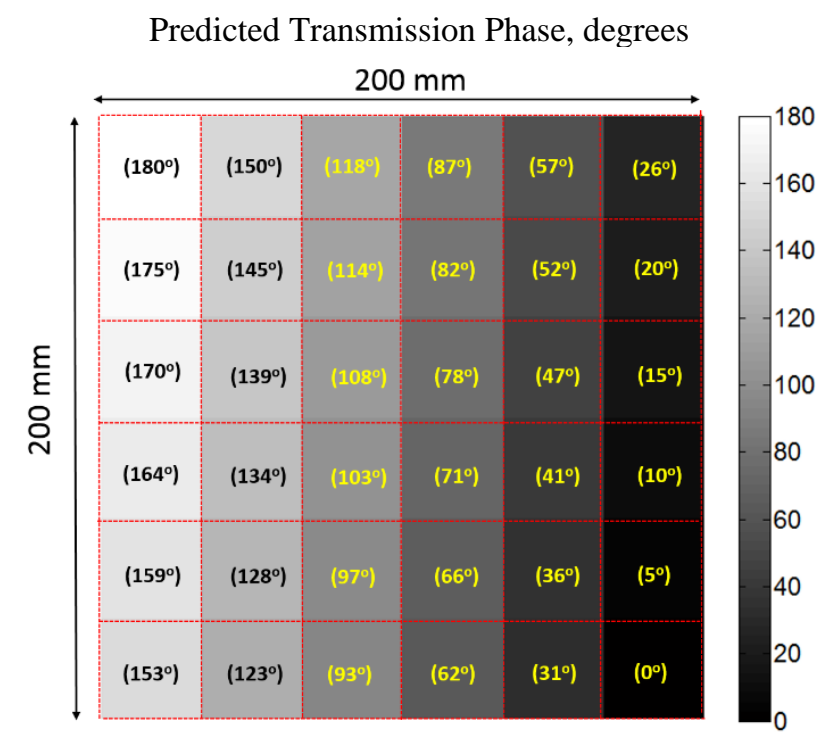

(c)

Figure 10: Example design used to validate the design methodology. (a) A $200 \mathrm{~mm} \times 200 \mathrm{~mm} \times 8 \mathrm{~mm}$ plate divided into a 6 by 6 grid of cells. Within each cell are shown the order, $N$, of the space-filling curve and the predicted effective permittivity. (b) Fabricated part after FDM printing. (c) Predicted and $(d)$ measured transmission phases averaged over each cell when illuminated by a $30 \mathrm{GHz}$ plane wave.

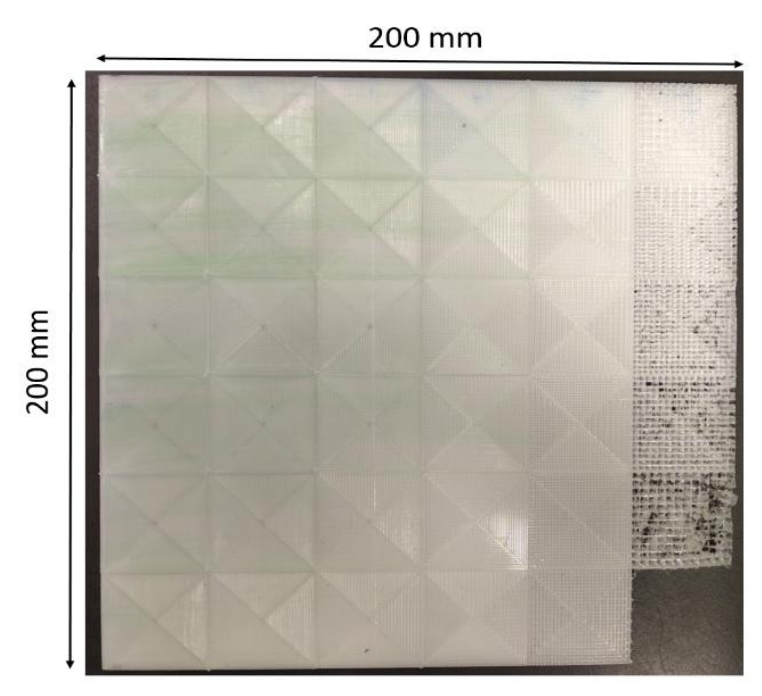

(b)

Measured Transmission Phase, degrees

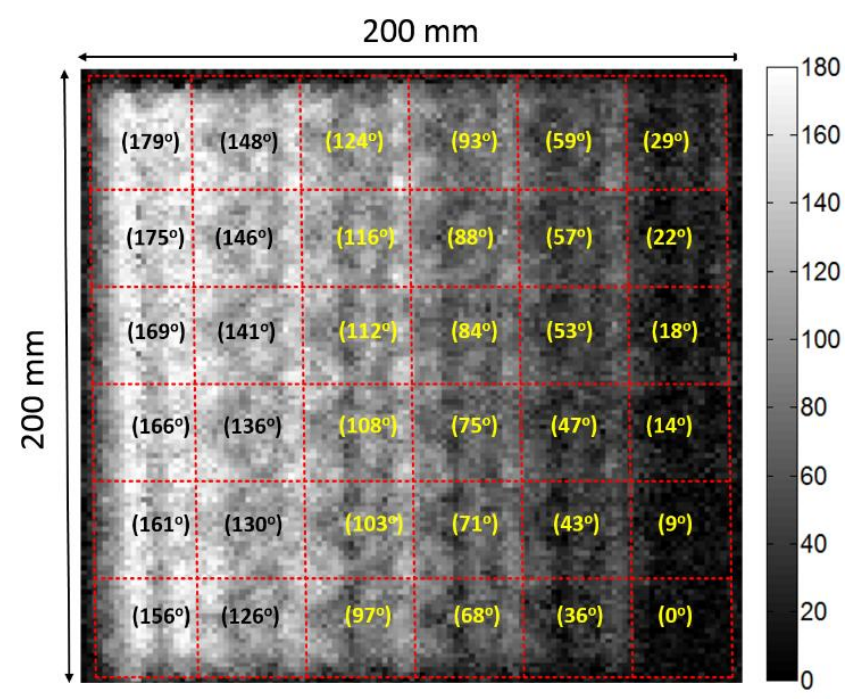

(d) 


\subsection{Example \#2: Graded Index Lens}

As a practical example, a graded index flat lens (GRIN) was designed and fabricated for use at microwave frequencies. The lens was designed to focus a $30 \mathrm{GHz}$ plane wave with a focal length of $F_{1}=680 \mathrm{~mm}$. The diameter of the lens was set to $\mathrm{R}=150 \mathrm{~mm}$. The lens thickness, $\mathrm{d}=8 \mathrm{~mm}$, was chosen to produce a $180^{\circ}$ phase variation at $30 \mathrm{GHz}$ with maximum permittivity at the center of the lens of $\varepsilon_{\mathrm{r}}(0)=2.68$. Using an approximate expression to design GRIN lenses, the authors determined the desired permittivity distribution as a function of the radial distance, $r$, from the center of the lens to be

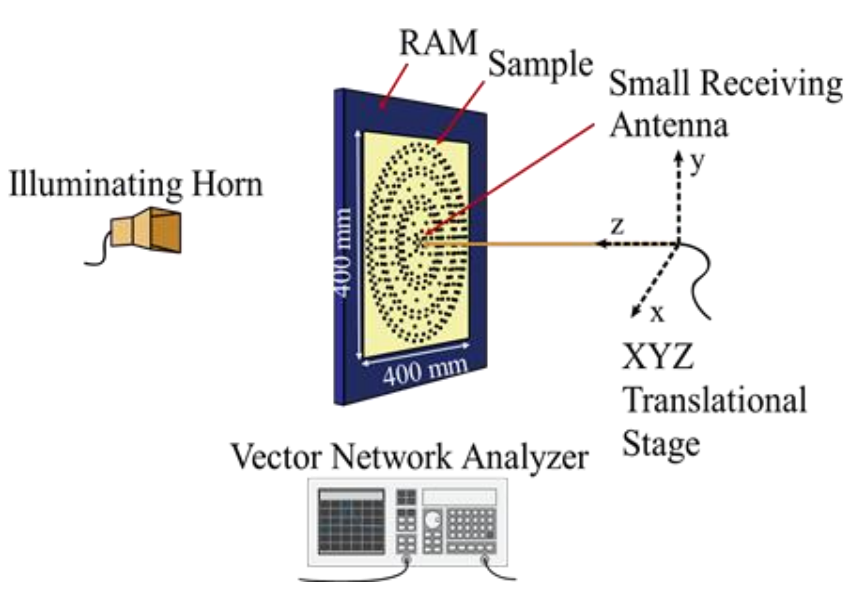

Figure 11. Characterization system used to measure plane wave transmission through samples.

$$
\varepsilon_{r}(r)=\left[\sqrt{\varepsilon_{r}(0)}-\sqrt{F_{l}^{2}+r^{2}}-\frac{F_{l}}{d}\right]^{2}
$$

Using (4), the permittivity distribution was calculated (Fig. 12a). Then, the algorithm described in Section 2.3 was used to design a space-filling curve geometry that best approximated this continuous permittivity distribution. For this example, the unit cell size, $\Lambda$, was fixed at $4.0 \mathrm{~mm}$. As in previous examples, the lens was FDM printed with a polycarbonate filament with an extruded diameter and layer thickness of $0.3 \mathrm{~mm}$ and $0.125 \mathrm{~mm}$, respectively. The final fabricated part and its micro-CT scans showing the printed microstructure are shown in Figure 12b.

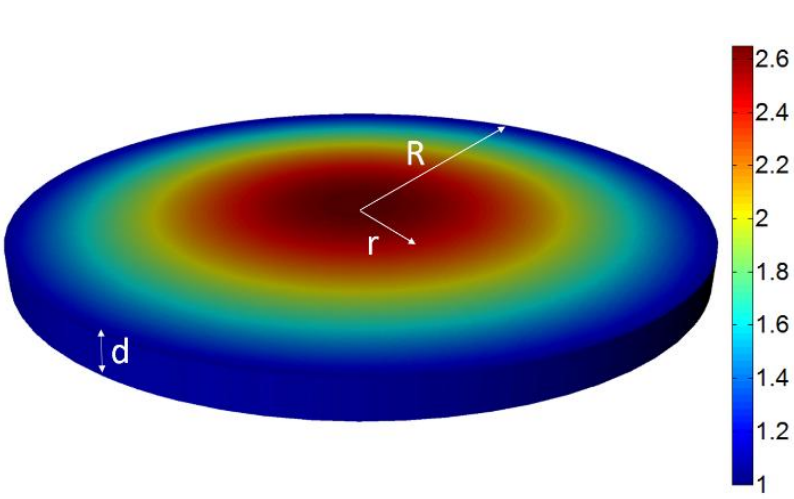

(a)

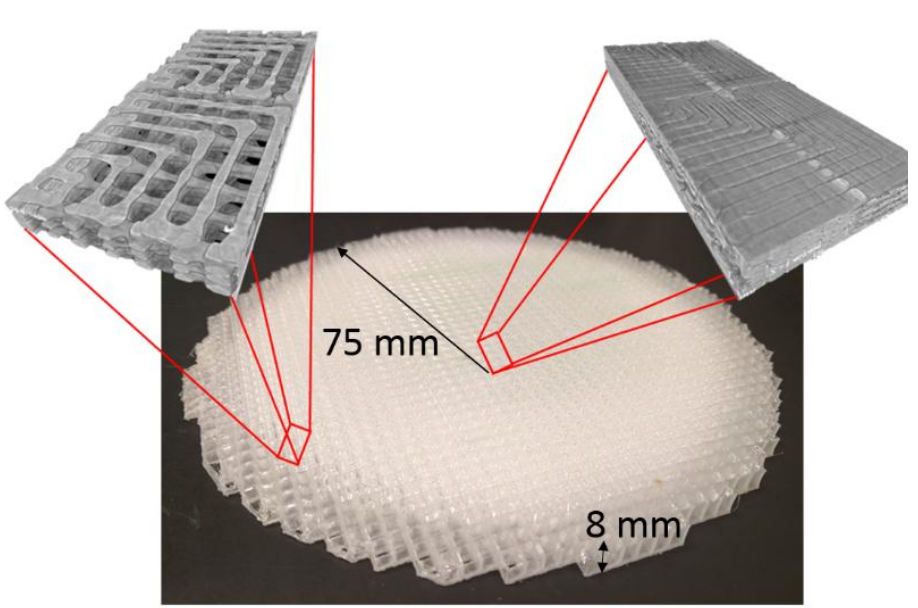

(b)

Figure 12. (a) Desired permittivity distribution for graded index lens. (b) Printed graded index lens. The magnified images were obtained using microCT imaging and show the space-filling curve geometry at two different locations on the lens surface.

To experimentally validate this fabricated GRIN lens, the authors used the characterization system shown in Figure 11. The part was illuminated with a normally incident $30 \mathrm{GHz}$ plane wave. To 
measure the focused energy, a small antenna probe scanned along the optical axis, and a vector network analyzer measured the complex transmission. Figure 13 shows the measured and predicted values for the normalized intensity along the optical axis. There was no data collected past $680 \mathrm{~mm}$ due to size limitation of the measurement chamber. While the two curves are quite similar in general shape, the focal distance is slightly shorter for the measured values $(625 \mathrm{~mm})$ than the designed values $(680 \mathrm{~mm})$. It is believed this results from errors in the permittivity distribution near the outer edges of the lens where the permittivity values are low.

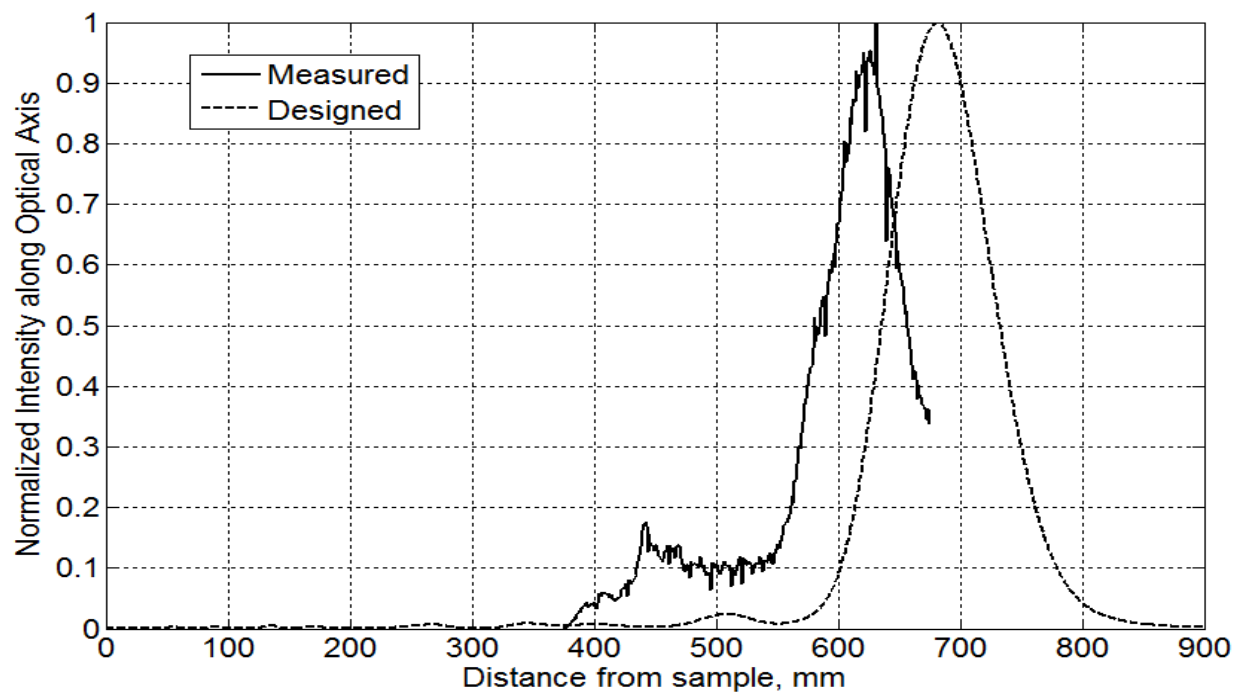

Figure 13: Designed and measured normalized intensity along the optical axis of the GRIN lens as a function of distance from the back surface of the sample. The lens was illuminated by a normally incident plane wave at $30 \mathrm{GHz}$.

\section{Discussion/Conclusion}

This paper describes a novel methodology to design and manufacture structures with graded electromagnetic properties by utilizing space-filling curve geometries. This approach offers three main advantages over previously published methods. Specifically, 1) the ability to fabricate mechanically robust graded structures without need for a support network, 2) the ability to print with very low electromagnetic loss polymers and 3) the capability of being conducive to FDM printing, the most prevalent and cost effective method available for additive manufacturing. The authors described and experimentally validated the computational approach to predicting the effective electromagnetic properties of these space-filling curves. The researchers also demonstrated the successful application of the design approach with two examples, a simple grid of varying permittivity cells and a graded index lens that focused energy at microwave frequencies. The authors are currently extending this new design and fabrication methodology to a wide range of other applications, including graded index lens antennas and passive beam forming structures. 


\section{Funding Acknowledgement}

This work was supported by the Office of Naval Research.

\section{References}

1. Good, Brandon, et al. "Design of graded index flat lenses with integrated antireflective properties", Microwave and Optical Technology Letters, 54.12 (2012): 2774-2781.

2. Ransom, P., et al. "Fabrication of wideband antireflective coatings using fused deposition modelling", Electronics Letters, 52.5 (2016): 352-354.

3. Grann, E., Moharam, M., and Pommet, D.: "Optimal design for anti-reflective tapered twodimensional subwavelength grating structures", JOSA A, 12, (2), 1995, pp. 333-339

4. Grann, E., Moharam, M., and Pommet, D.: "Artificial uniaxial and biaxial dielectrics with use of two-dimensional subwavelength binary gratings", JOSA A, 11, (10), 1994, pp. 2695-2703

5. Rumpf, Raymond C., et al. "3D printed lattices with spatially variant self-collimation", Progress in Electromagnetics Research", 139 (2013): 1-14.

6. Liang, Min, et al. "A 3-D Luneburg lens antenna fabricated by polymer jetting rapid prototyping", IEEE Transactions on Antennas and Propagation, 62.4 (2014): 1799-1807.

7. Deffenbaugh, Paul I., Raymond C. Rumpf, and Kenneth H. Church. "Broadband microwave frequency characterization of 3-D printed materials", IEEE Transactions on Components, Packaging and Manufacturing Technology, 3.12 (2013): 2147-2155.

8. Peano, G., "Sur une courbe, qui remplit toute une aire plane", Mathematische Annalen 36 (1): (1890), 157-160

9. Hilbert, D., "Ueber die stetige Abbildung einer Line auf ein Flächenstück", Mathematische Annalen 38 (3): (1891), 459-460

10. Regazzoni, Carlo S. and Andrea Teschioni. "A new approach to vector median filtering based on space filling curves", IEEE Transactions on Image Processing, 6.7 (1997): 1025-1037.

11. A. Sihvola, "Generalized Mixing Rules", in Electromagnetic Mixing Formulas and Applications, London, United Kingdom, IET Electromagnetic Waves Series, 47, (2008)

12. Moharam M. G., Grann E.B., Pommet D.A. and Gaylord T.K., "Formulation for stable and efficient implementation of the rigorous coupled wave analysis of binary gratings", J. Opt. Soc. Am. A, vol. 12, (1995); 1068-1076.

13. Castante A., Mercier J. and Maurel F., "Generalized method for retrieving effective parameters of anisotropic metamaterials”, Optics Express, 22.24 (2014) 